\title{
BOILING-STEAMING GALACTIC DISK: VERTICAL DUST JETS IN THE DISK-HALO INTERFACE
}

\author{
Y.SOFUE ${ }^{1}$, K.WAKAMATSU ${ }^{2}$, and D.F.MALIN ${ }^{3}$ \\ 1.Institute of Astronomy, University of Tokyo, Japan \\ 2.Physics Department, Gifu University, Japan \\ 3.Anglo-Australian Observatory, Australia
}

\begin{abstract}
Optical photographs of highly-tilted, dust-rich nearby spiral galaxies like NGC253 have revealed numerous vertical dark filaments which we call vertical dust jets (VDJ). The VDJ exdend more than a few kpc from the disk in an almost coherent manner, while they are as thin as a few tens of pc. They are most likely due to boiling-steaming galactic disk, which ejects gas into the halo. The coherency suggests that VDJ trace large-scale poloidal magnetic lines of force.
\end{abstract}

\section{INTRODUCTION}

Vertically extending filamentary structures are often seen in optical photographs of tilted galaxies. The vertical filaments may provide information about the largescale circulation of gas from the disk into halo and vice versa. They may also trace vertical magnetic fields (Sofue 1987). We present result of a systematic study of vertical filaments based on un-sharp masked optical photographs of nearby, dustrich galaxies.

\section{PHOTOGRAPHS}

We have undertaken optical imaging of the nearby galaxis, NGC 253, NGC 4945, NGC 4594, and NGC 1808. The photographs have been taken with the AAT 4-m telescope and the Las Campanas 2-m telescope. The plates have been unsharp masked, so that faint, filamentary structures are enhanced.

NGC 253 - Central Region: Vertical dust lanes most extensively emerge from the central 1-2 kpc region. The jets are as long as a few minutes of arc, or $2-3$ kpc above the galactic plane, while their widths are as narrow as a few arc sec (a few tens of pc). Fig. 1 shows a sketch of the vertical dust jets in NGC 253.

$N G C 253$ - Outer Disk: Vertical dust lanes are also well found in the outer disk at galacto-centric distances up to 6-8 kpc. Their sizes are comparable to those found in the central region. However, the outer dust lanes are more complicated, more disturbed, and look something like steam from a hot dish.

$N G C$ 4945: This is also a dust-rich galaxy, showing complicated dusty filaments emerging from the disk. They are not well aligned as in NGC 253. The vertical extents of the filaments are estimated to be an order of magnitude larger than the 


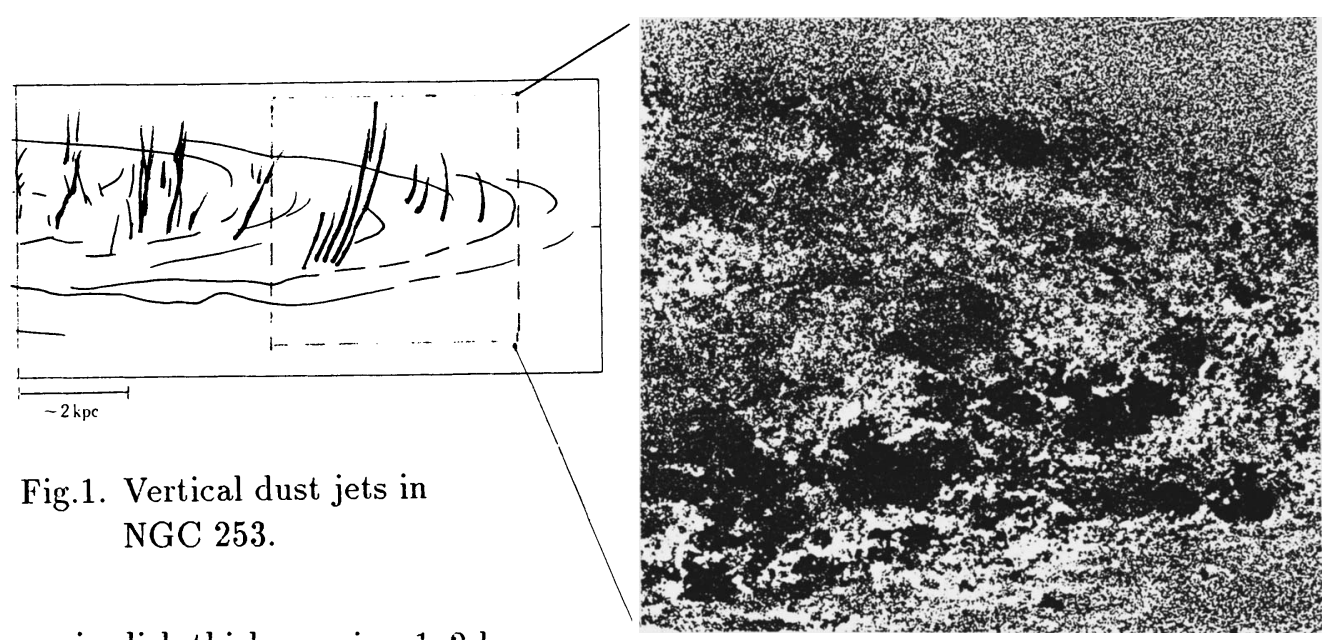

main disk thickness, i.e. $1-2 \mathrm{kpc}$.

$N G C$ 4594: This is an almost edge-on Sc galaxy. Dust is well confined in a thin galactic layer. However, here we can also see many dust filaments emerging almost perpendicular to the disk plane for about $1 \mathrm{kpc}$.

NGC 1808: The central region shows jet-like dust lanes, well collimated, opening, and extend for a few kpc. [For more other galaxies, see Sofue (1987)]

\section{BOILING-STEAMING GALACTIC DISK}

The VDJ phonomenon seems common in any disk galaxies, in so far as we have examined published optical photographs (Sofue 1987). The VDJ phenomenon is most probably manifestation of ejection of dusty interstellar matter from the galactic plane into halo. We propose the following models to explain VDJ:

Boiling-Steaming Disk: Galactic gas disks are "boiling". The energy sources are supernova explosions, stellar winds, and inflating magnetic bubbles with cosmic rays. The boiling disk should then give "steam" (vapor) toward the halo, similarly to a hot plate. In the halo the steam will cool down and become molecular gas. Radiation pressure by star light on dust grains will also act as a driving force (Ferrini et al 1990).

Vertical Magnetic Fields: The very coherent alignment of the dust jets suggests that they trace some guid lines, which are likely poloidal magnetic lines of force (Sofue and Fujimoto 1987).

\section{references}

Ferrini, et al 1990, in this issue.

Sofue, Y. 1987, Publ. Astron. Soc. Japan, 39, 547

Sofue, Y., and Fujimoto, M. 1987, Publ. Astron. Soc. Japan, 39, 843 